\title{
On a generalization of the Perron integral on one-dimensional intervals
}

\author{
by Jaroslav Kurzweil and Jiří Jarní (Prague)
}

Zdzislaw Opial in memoriam

\section{Introduction}

The integral studied in the present paper is a generalization of the one-dimensional Perron integral. We call it an $\mathrm{H}$-integral and denote by (H) $\int_{a}^{b} f d t$, where $H$ specifies a system of pointed intervals used in the definition. The integral is defined as a certain limit of the sums

$$
S(f, \Delta)=\sum_{j=1}^{k} f\left(t_{j}\right)\left(x_{j}-x_{j-1}\right),
$$

where $x_{0}<a=t_{1}<x_{1}<t_{2}<\ldots<x_{k-1}<t_{k}=b<x_{k}$. The intervals $\left[x_{j-1}\right.$, $\left.x_{j}\right]$ forming a covering (rather than a partition) of the interval $[a, b]$, the integral has some unexpected properties. For example, it is possible that $(H) \int_{a}^{b} f d t$ exists but $(H) \int_{a}^{c} f d t$ does not for some $c \in(a, b)$. For some choices of the set $H$ we have $(H) \int_{-1}^{1} d x / x=0$.

In our paper [4], such examples were presented and the transformation of the integral for a special choice of the set $H$ was discussed. In the general case, the main results were given without proofs. The aim of the present paper is to give brief proofs of the results announced in [4], Section 3.

\section{Preliminaries}

Let $[a, b]$ be an interval. Then any linite set

$$
\Delta=\left\{\left(t_{j},\left[x_{j-1}, x_{j}\right]\right) ; j=1, \ldots, k\right\}
$$


such that

$$
x_{0}<t_{1}=a<x_{1}<t_{2}<\ldots<t_{k-1}<x_{k-1}<t_{k}=b<x_{k}
$$

is called a covering of $[a, b]$.

If $\delta$ is a gauge on $[a, b]$, i.e., $\delta:[a, b] \rightarrow(0,+\infty)$, and

$$
\left[x_{j-1}, x_{j}\right] \subset B\left(t_{j}, \delta\left(t_{j}\right)\right), \quad j=1, \ldots, k,
$$

then $\Delta$ is said to be $\delta$-fine. (Here and in the sequel, $B(t, r)$ $=(t-r, t+r)$.)

Write

$$
\begin{aligned}
J & =J[a, b]=\{(t,[x, y]) ; t \in[a, b], x<t<y\}, \\
\operatorname{Sym} & =\operatorname{Sym}[a, b]=\left\{(t,[x, y]) \in J ; t=\frac{1}{2}(x+y)\right\} .
\end{aligned}
$$

Let $H=H[a, b]$ be a set such that Sym $\subset H \subset J$ and

(1) for every $(t,[x, y]) \in H$ there is $\xi>0$ such that $(t,[x+h, y-h]) \in H$ for every $h,|h|<\xi$.

A covering $\Delta$ such that $\Delta \subset H$ will be called an $H$-covering.

1.1. Remark. Let $K>0, \varrho \geqslant 1$ be constants. Then $A S_{K . e}=\left\{(t,[x, y]) \in J ; 0<t-x<y-t+K(y-t)^{\varrho}, 0<y-t<t-x+K(t-x)^{\varrho}\right\}$ satisfies Sym $\subset A S_{K, \varrho} \subset J$ and has property (1). (Cf. [4], Note 3.2.)

1.2. Remark. Given a set

$$
\Xi=\left\{\left(\tau_{i},\left[\xi_{i-1}, \xi_{i}\right]\right) ; i=1, \ldots, l\right\} \subset H
$$

and a gauge $\delta$ on $\left[\tau_{1}, \tau_{l}\right]$ such that

$$
\xi_{0}<\tau_{1}<\xi_{1}<\tau_{2}<\ldots<\tau_{1-1}<\xi_{1-1}<\tau_{1}<\xi_{1}, \quad\left[\xi_{i-1}, \xi_{i}\right] \subset B\left(\tau_{i}, \delta\left(\tau_{i}\right)\right) \text {; }
$$

then there exists $\eta>0$ such that

$$
\begin{gathered}
\xi_{0}+h<\tau_{1}<\xi_{1}-h<\tau_{2}<\ldots<\tau_{l-1}<\xi_{l-1}(-1)^{l-1} h<\tau_{l}<\xi_{l}+(-1)^{l} h, \\
{\left[\xi_{i-1}+(-1)^{i-1} h, \xi_{i}+(-1)^{i} h\right] \subset B\left(\tau_{i}, \delta\left(\tau_{i}\right)\right)}
\end{gathered}
$$

for $i=1, \ldots, l$ provided $|h|<\eta$. (This follows from (1) and from the fact that $\Xi$ is finite.)

The set

$$
\Xi_{h}=\left\{\left(\tau_{i},\left[\xi_{i-1}+(-1)^{i-1} h, \xi_{i}+(-1)^{i} h\right]\right) ; i=1, \ldots, l\right\}
$$

will be called an h-modification (or briefly a modification) of the set $\Xi$.

In particular, if $\Xi$ is a $\delta$-fine $H$-covering of $[a, b]$, then its $h$-modification 
(with $h$ sufficiently small) is a $\delta$-fine $H$-covering of $[a, b]$ as well. This fact will be frequently used in the proofs throughout the paper without further notice.

\section{Definition and main properties of the $H$-integral}

2.1. Definition. A function $f:[a, b] \rightarrow R$ is called $H$-integrable (on $[a, b]$ ) if there is $q \in R$ such that for every $\varepsilon>0$ there is a gauge $\delta$ on $[a, b]$ such that

$$
|q-S(f, \Delta)|<\varepsilon
$$

for every $\delta$-fine $H$-covering $\Delta$ of $[a, b]$, where

$$
S(f, \Delta)=\sum_{j=1}^{k} f\left(t_{j}\right)\left(x_{j}-x_{j-1}\right) .
$$

The number $q$ is the $H$-integral of $f$ over $[a, b]$ and we write

$$
q=(H) \int_{a}^{b} f(t) d t=(H) \int_{a}^{b} f d t
$$

2.2. Remark. If $H=J$, then the $H$-integral is the Perron integral. Indeed, let $f:[a, b] \rightarrow R, \gamma \in R$. It is well known (cf. [2], Section 1.2; [3], Theorem 3.5; [1], Appendix A, Proposition 4.3) that the Perron integral of $f$ exists and $\gamma=(P) \int_{a}^{b} f d t$ iff for every $\varepsilon>0$ there exists such a gauge $\delta$ on $[a, b]$ that

$$
\left|\gamma-\sum_{j=1}^{k} f\left(t_{j}\right)\left(x_{j}-x_{j-1}\right)\right| \leqslant \varepsilon
$$

holds for every sequence

$$
a=x_{0} \leqslant t_{1} \leqslant x_{1} \leqslant \ldots \leqslant x_{k-1} \leqslant t_{k} \leqslant x_{k}=b
$$

satisfying

$$
t_{j}-\delta\left(t_{j}\right)<x_{j-1}, \quad x_{j}<t_{j}+\delta\left(t_{j}\right) .
$$

It can be assumed without loss of generality that $a<t-\delta(t), t+\delta(t)<b$ for $t \in(a, b)$, so that we have in addition $t_{1}=a, t_{k}=b$. It is not difficult to prove that the same concept of integral is obtained if $(*)$ is replaced by

$$
x_{0}<a=t_{1}<x_{1}<\ldots<x_{k-1}<t_{k}=b<x_{k},
$$

and this modified concept is at the same time the $H$-integral for $H=J$.

2.3. Proposition. A function $f$ is $H$-integrable if and only if for every $\varepsilon>0$ there is a gauge $\delta$ such that

$$
\left|S\left(f, \Delta_{1}\right)-S\left(f, \Delta_{2}\right)\right|<\varepsilon
$$

for any two $\delta$-fine $H$-coverings $\Delta_{1}, \Delta_{2}$.

Proof is standard. 
2.4. Remark. Let $\delta$ be a gauge on $[a, \mathrm{~b}]$, let $d \in(a, b)$. The point $d$ is said to be $\delta$-reachable from a (more precisely, $\delta$-H-reachable from $a$ ) if there is a set

$$
\theta=\left\{\left(\sigma_{i},\left[\vartheta_{i-1}, \vartheta_{i}\right]\right) ; i=1, \ldots, m\right\} \subset H
$$

such that

$$
\begin{gathered}
\vartheta_{0}<\sigma_{1}=a<\vartheta_{1}<\sigma_{2}<\ldots<\vartheta_{m-1}<\sigma_{m}<\vartheta_{m}=d, \\
{\left[\vartheta_{i-1}, \vartheta_{i}\right] \subset B\left(\sigma_{i}, \delta\left(\sigma_{i}\right)\right) .}
\end{gathered}
$$

(Notice that $\theta$ is a covering of $\left[a, \sigma_{m}\right]$ but not of $[a, d]$.)

Similarly, $d$ is called $\delta$-reachable from $b$ if $\theta$ satisfies (2) and

$$
d=\vartheta_{0}<\sigma_{1}<\vartheta_{1}<\sigma_{2}<\ldots<\vartheta_{m-1}<\sigma_{m}=b<\vartheta_{m} .
$$

The set $\theta$ will be called a $\delta$-chain from a to $d$ (or, as the case may be, from $b$ to $d$ ). Lemma 2.4 [4] asserts that the set of points $d \in(a, b)$ which are not $\delta$-reachable from either $a$ or $b$ is at most countable.

Indeed, let $s$ be the supremum of all $c \in(a, b)$ such that in $(a, c)$ there are at most countably many points not $\delta$-reachable from $a$. We have $s \geqslant a+\delta(a)$ since every $x \in(a, a+\delta(a))$ is $\delta$-reachable from $a$ (it suffices to put $\vartheta_{0}=2 a-$ $\left.x<\sigma_{1}=a<\vartheta_{1}=x\right)$. Assume $s<b$. Then in the interval $(s, \min (s+\delta(s), b))$ there exist uncountably many points not $\delta$-reachable from $a$. Let $x$ be such a point. Then $y=s-(x-s)=2 s-x$ cannot be $\delta$-reachable from $a$ since otherwise we could extend the corresponding chain from $a$ to $y$ by the pair $(s,[2 s-x, x]) \in$ Sym, thus obtaining a $\delta$-chain from $a$ to $x$. But there are only countably many points $y<s$ not $\delta$-reachable from $a$, which is a contradiction.

2.5. Remark. It follows from [4], Lemma 2.3 or from Remark 2.4 above that for every gauge $\delta$ there exists a $\delta$-fine $H$-covering. In fact, there always exists a $\delta$-fine Sym-covering. Indeed, by Remark 2.4 we can find a point $d, b-\delta(b)<d<b$, which is $\delta$-Sym-reachable from $a$. By adding the element $(b,[d, 2 b-d])$ to the corresponding chain we obtain a $\delta$-fine Sym-covering of $[a, b]$.

2.6. TheOREM. Let $f:[a, b] \rightarrow R$ be H-integrable. Denote by $E=E_{f}$ the set of all $c \in(a, b)$ such that $\left.f\right|_{[a, c]}$ is not $H$-integrable. Then $E$ is at most countable.

Proof. Set $\varepsilon_{j}=2^{-j}$ and find the corresponding gauge $\delta_{j}$ from the definition of the $H$-integral $(H) \int_{a}^{b} f d t$. Denote by $W_{j}$ the set of all points $c \in(a, b)$ which are not $\delta_{j}$-reachable from either $a$ or $b$, and put $W=\bigcup_{j=1}^{\infty} W_{j}$. By Remark 2.4 the set $W$ is at most countable.

Let $c \in(a, b) \backslash W$. Then $(H) \int_{a}^{c} f d t$ exists.

Indeed, let $\varepsilon>0$. Find $j$ such that $\frac{1}{2} \varepsilon>2^{-j}$. Since $c$ is $\delta_{j}$-reachable from 
$b$, there exists a set

$$
\theta=\left\{\left(\sigma_{i},\left[u_{i-1}, u_{i}\right]\right) ; i=1, \ldots, m\right\} \subset H
$$

such that

$$
\begin{gathered}
c=u_{0}<\sigma_{1}<u_{1}<\sigma_{2}<\ldots<\sigma_{m-1}<u_{m-1}<\sigma_{m}=b<u_{m}, \\
{\left[u_{i-1}, u_{i}\right] \subset B\left(\sigma_{i}, \delta_{j}\left(\sigma_{i}\right)\right) .}
\end{gathered}
$$

For this set, find $\eta>0$ such that its every $h$-modification with $0<h<\eta$ is a $\delta_{j}$-fine $H$-covering of $\left[\sigma_{1}, b\right]$ (cf. Remark 1.2).

Further, choose $h, 0<h<\eta$, so that

$$
2 h \sum_{i=1}^{m}\left|f\left(\sigma_{i}\right)\right|<2^{-j}
$$

and a gauge $\tilde{\delta}_{j}$ such that

$$
\begin{array}{ll}
\delta_{j}(t)<\min \left(\delta_{j}(t), c-t\right) & \text { for } a \leqslant t<c, \\
\delta_{j}(c)<\min \left(h, \delta_{j}(c)\right), & \\
\delta_{j}(t)=\delta_{j}(t) & \text { for } c<t \leqslant b .
\end{array}
$$

Let $\Delta^{1}, \Delta^{2}$ be $\delta_{j}$-fine $H$-covering of $[a, c]$ where

$$
\Delta^{p}=\left\{\left(t_{j}^{p},\left[x_{j-1}^{p}, x_{j}^{p}\right]\right) ; j=1, \ldots, k_{p}\right\}, \quad p=1,2 .
$$

Write $x_{p}=x_{k_{p}}^{p}-c$; then $0<x_{p}<h$. Construct $x_{p}$-modifications of the set $\theta$ for $p=1,2$, and denote them by $\theta^{1}, \theta^{2}$. By the choice of $h$ and $\tilde{\delta}_{j}$, the sets $\Delta^{p} \cup \theta^{p}, p=1,2$, are $\delta_{j}$-fine $H$-covering of $[a, b]$. Evidently,

$$
S\left(f, \Delta^{p} \cup \theta^{p}\right)=S\left(f, \Delta^{p}\right)+S\left(f, \theta^{p}\right)
$$

and

$$
\begin{aligned}
\left|S\left(f, \theta^{1}\right)-S\left(f, \theta^{2}\right)\right| & =\mid \sum_{i=1}^{m} f\left(\sigma_{i}\right)\left[\left(u_{i}+(-1)^{i} \varkappa_{1}-u_{i-1}-(-1)^{i-1} \varkappa_{1}\right)\right. \\
& \left.-\left(u_{i}+(-1)^{i} \varkappa_{2}-u_{i-1}-(-1)^{i-1} \varkappa_{2}\right)\right] \mid \\
& \leqslant \sum_{i=1}^{m}\left|f\left(\sigma_{i}\right)\right| 2\left(\varkappa_{1}-\varkappa_{2}\right) \leqslant 2 h \sum_{i=1}^{m}\left|f\left(\sigma_{i}\right)\right|<\frac{1}{2} \varepsilon .
\end{aligned}
$$

Consequently, we have

$$
\left|S\left(f, \Delta^{1}\right)-S\left(f, \Delta^{2}\right)\right| \leqslant\left|S\left(f, \Delta^{1} \cup \theta^{1}\right)-S\left(f, \Delta^{2} \cup \theta^{2}\right)\right|+\left|S\left(f, \theta^{1}\right)-S\left(f, \theta^{2}\right)\right|<\varepsilon,
$$

and the desired integrability (over $[a, c]$ ) follows by Proposition 2.3.

2.7. TheOREM. Let $a<c<b$ and let two of the integrals in the equality

$$
(H) \int_{a}^{c} f d t+(H) \int_{c}^{b} f d t=(H) \int_{a}^{b} f d t
$$


exist. Then the third integral exists as well and the equality holds.

Proof. Consider the case where the first and the last integral exist. Let $\varepsilon>0$, and find gauges $\delta_{1}, \delta_{2}$ on $[a, c],[a, b]$, respectively, corresponding to $\varepsilon$ in the sense of Definition 2.1. Without loss of generality we can and will assume that

$$
\begin{aligned}
& \delta_{2}(t) \leqslant \delta_{1}(t) \quad \text { for } t \in[a, c], \\
& \delta_{2}(t) \leqslant|t-c| \quad \text { for } t \in[a, b] \backslash\{c\}, \\
& 2|f(c)| \delta_{2}(c)<\varepsilon .
\end{aligned}
$$

Let $\Delta$ be a $\delta_{2}$-fine $H$-covering of $[c, b]$, where

$$
\Delta=\left\{\left(t_{j},\left[x_{j-1}, x_{j}\right]\right) ; j=1, \ldots, k\right\} \text {. }
$$

Let $h>0$ be such that the $h$-modification $\Delta_{h}$ of $\Delta$ is a $\delta_{2}$-fine $H$-covering of $[c, b]$, and $x_{0}+h$ is $\delta_{2}$-reachable from $a$. (Existence of such an $h$ follows from Remarks 1.2 and 2.4.) Moreover, let $h$ be so small that

$$
2 h \sum_{j=1}^{k}\left|f\left(t_{j}\right)\right|<\varepsilon
$$

Let $\theta$ be the first set from Remark 2.4 with $d=x_{0}+h$ and $\delta=\delta_{2}$. Then the set $\theta \cup \Delta_{h}$ is a $\delta_{2}$-fine $H$-covering of $[a, b]$, and the set $\theta \cup\left\{\left(c,\left[x_{0}+h, x_{1}-h\right]\right)\right\}$ is a $\delta_{1}$-fine $H$-covering of $[a, c]$. Consequently,

$$
\begin{aligned}
\left|S(f, \Delta)-(H) \int_{a}^{b} f d t+(H) \int_{a}^{c} f d t\right| & \leqslant\left|S(f, \Delta)-S\left(f, \Delta_{h}\right)\right| \\
+ & \left|S\left(f, 0 \cup\left\{\left(c,\left[x_{0}+h, x_{1}-h\right]\right)\right\}\right)-(H) \int_{a}^{c} f d t\right| \\
+ & |f(c)|\left(x_{1}-h-x_{0}-h\right)+\left|S\left(f, \theta \cup \Delta_{h}\right)-(H) \int_{a}^{b} f d t\right| \\
& \leqslant 2 h \sum_{j=1}^{k}\left|f\left(t_{j}\right)\right|+\varepsilon+2|f(c)| \delta_{2}(c)+\varepsilon<4 \varepsilon
\end{aligned}
$$

which proves the existence of the integral $(H) \int_{c}^{b} f d t$ as well as the validity of equality (3).

Proofs of the other cases are analogous.

2.8. Remark. Let $(H) \int_{a}^{b} f d t$ exist, let $c, d \in[a, b], c<d$. If $c, d \in[a, b] \backslash E$, then by Theorems 2.6, 2.7 the integral $(H) \int_{c}^{d} f d t$ exists. Conversely, if $(H) \int_{c}^{d} f d t$ exists, then by Theorem 2.6 there is $\tau \in(c, d)$ such that the integrals $(H) \int_{a}^{\tau} f d t$ 
and $(H) \int_{c}^{\tau} f d t$ exist, and by Theorem 2.7 we obtain $c \notin E$ and similarly $d \notin E$.

\section{Indefinite $H$-integral and its properties}

In this section let $f:[a, b] \rightarrow R$ be $H$-integrable, and define

$$
F(a)=0, \quad F(t)=(H) \int_{a}^{t} f(s) d s \quad \text { for } t \in[a, b] \backslash E,
$$

where $E$ is the (at most countable, cf. Theorem 2.6) set of points $c \in(a, b)$ such that $(H) \int_{a}^{c} f d t$ does not exist.

3.1. THEOREM. The function $F$ is continuous on $[a, b] \backslash E$.

Proof. Let $c \in[a, b] \backslash E$, let $\left(c_{n}\right)$ be an increasing sequence, $c_{n} \notin E$, $\lim c_{n}=c$. Let $\varepsilon>0$. Find the gauge $\delta$ on $[a, c]$ corresponding to the $n \rightarrow \infty$

definition of the integral $(H) \int_{a}^{c} f d t$, assuming without loss of generality that

$$
\delta(t)<c-t \quad \text { for } t<c, \quad|f(c)| \delta(c)<\varepsilon .
$$

Let $k$ be such an integer that $c_{k}>c-\delta(c)$. Let $\delta_{k}$ be the gauge from Definition 2.1 corresponding to $\varepsilon$ and $(H) \int_{a}^{c_{k}} f d t$. Without loss of generality let us assume that $\delta_{k}(t) \leqslant \delta(t)$ for $t \in\left[a, c_{k}\right]$. Let $\Delta$ be a $\delta_{k}$-fine $H$-covering of $\left[a, c_{k}\right]$ whose last element is $\left(c_{k},\left[x_{k-1}, x_{k}\right]\right)$. We have $x_{k}<c$ by (4). Hence $\Delta \cup$ $\left\{\left(c,\left[x_{k}, 2 c-x_{k}\right]\right)\right\}$ is a $\delta$-fine covering of $[a, c]$. Consequently,

$$
\begin{aligned}
\left|(H) \int_{a}^{c} f d t-(H) \int_{a}^{c_{k}} f d t\right| \leqslant\left|(H) \int_{a}^{c} f d t-S\left(f, \Delta \cup\left\{\left(c,\left[x_{k}, 2 c-x_{k}\right]\right)\right\}\right)\right| & \\
& +|f(c)| 2 \delta(c)+\left|S(f, \Delta)-(H) \int_{a}^{c_{k}} f d t\right|<3 \varepsilon,
\end{aligned}
$$

which proves the theorem.

Before formulating a converse result, we will prove the version of the Saks-Henstock lemma corresponding to the $H$-integral.

3.2. TheOREM (Saks-Henstock lemma). Let $f:[a, b] \rightarrow R$ be $H$-integrable, $\varepsilon>0$. Let $\delta$ be the gauge from Definition 2.1 and let

$$
\left\{\left(t_{j},\left[u_{j}, v_{j}\right]\right) ; j=1, \ldots, k\right\} \subset H
$$

satisfy

$$
\begin{gathered}
{\left[u_{j}, v_{j}\right] \subset B\left(t_{j}, \delta\left(t_{j}\right)\right)} \\
a \leqslant u_{1}<t_{1}<v_{1} \leqslant u_{2}<t_{2}<\ldots<v_{k-1} \leqslant u_{k}<t_{k}<v_{k} \leqslant b
\end{gathered}
$$


and $u_{j}, v_{j} \notin E$ for $j=1, \ldots, k$. Then

$$
\sum_{j=1}^{k}\left((H) \int_{u_{j}}^{v_{j}} f d t-f\left(t_{j}\right)\left(v_{j}-u_{j}\right)\right) \leqslant \varepsilon .
$$

Proof. Let us first make two remarks. First, we may and will assume, without loss of generality, that all inequalities in (7) are strict. Indeed, we can pass from the points $u_{j}, v_{j}$ to $u_{j}^{\prime}>u_{j}, v_{j}^{\prime}<v_{j}$ so that conditions (5)-(7) are fulfilled with the new points, and the "error" made by replacing $u_{j}, v_{j}$ by $u_{j}^{\prime}, v_{j}^{\prime}$ on the left-hand side of (8) is arbitrarily small. Second, notice that by Remark 2.8 the $H$-integrals of $f$ over $\left[u_{j}, v_{j}\right]$ and $\left[v_{j}, u_{j+1}\right]$ exist for $j=1, \ldots, k(k-1$, respectively).

Since the proof of Theorem 3.2 is technically rather complicated, we first prove a lemma.

3.3. Lemma. Let $\delta, t_{j}, u_{j}, v_{j}$ be from Theorem 3.2, let $\varrho>0$. Then there exists a $\delta$-fine $H$-covering $\Omega$ of $[a, b]$,

$$
\Omega=\left\{\left(\tau_{i},\left[\omega_{i-1}, \omega_{i}\right]\right) ; i=1, \ldots, l\right\}
$$

and integers $0<m_{1}<m_{2}<\ldots<m_{k}$ such that

$$
\begin{gathered}
\tau_{m_{j}}=u_{j}<\omega_{m_{j}}<\tau_{m_{j}+1}=t_{j}<\omega_{m_{j}+1}<v_{j}=\tau_{m_{j}+2}, \\
\omega_{m_{j}}-u_{j}=v_{j}-\omega_{m_{j}+1}<\varrho .
\end{gathered}
$$

Proof. Denote $s_{j}=\frac{1}{2}\left(v_{j}+u_{j+1}\right)$ for $j=1, \ldots, k-1, s_{k}=b$. Set

$$
\delta^{\prime}\left(s_{j}-\lambda\right)=\delta^{\prime}\left(s_{j}+\lambda\right)=\min \left(\delta\left(s_{j}-\lambda\right), \delta\left(s_{j}+\lambda\right)\right)
$$$$
\text { for } 0 \leqslant \lambda \leqslant s_{j}-v_{j}, j=1, \ldots, k-1,
$$

$\delta^{\prime}(t)=\delta(t) \quad$ otherwise, $t \in[a, b]$.

For $j=1, \ldots, k-1$ find $\sigma_{j}, 0<\sigma_{j}<\delta^{\prime}\left(s_{j}\right)$ such that

$$
v_{j}<s_{j}-\sigma_{j}<s_{j}+\sigma_{j}<u_{j+1} \text { and } s_{j}-\sigma_{j}, s_{j}+\sigma_{j} \notin E
$$

(cf. Theorem 2.6). Then there exists $h, 0<h<\varrho$, satisfying

$$
h<\xi\left(t_{j},\left[u_{j}, v_{j}\right]\right) \quad(\mathrm{cf} .(1)), h<s_{j}-\sigma_{j}-v_{j}, \quad h<\delta^{\prime}\left(u_{j}\right), \quad h<\delta^{\prime}\left(v_{j}\right)
$$

for $j=1, \ldots, k$, and such that the points $v_{j}+h$ are $\delta^{\prime}$-reachable from $s_{j}-\sigma_{j}$ and $u_{1}-h$ is $\delta^{\prime}$-reachable from $a$ (cf. Remark 2.4).

Now we will construct the desired covering.

By the choice of $h$ there exists a $\delta^{\prime}$-chain from $a$ to $u_{1}-h$; let it consist of points

$$
\omega_{0}, \tau_{1}=a, \omega_{1}, \ldots, \tau_{m_{1}-1}, \omega_{m_{1}-1}=u_{1}-h .
$$

Put $\tau_{m_{1}}=u_{1}, \omega_{m_{1}}=u_{1}+h, \tau_{m_{1}+1}=t_{1}, \omega_{m_{1}+1}=v_{1}-h, \tau_{m_{1}+2}=v_{1}, \omega_{m_{1}+2}$ $=v_{1}+h$. Again by the choice of $h$, the last point is $\delta^{\prime}$-reachable from $s_{1}-\sigma_{1}$. 
Suppose we have found the points of $\Omega$ up to a point $\omega_{m_{j}+2}=v_{j}+h$ in such a way that (9) is fulfilled. Then there is a $\delta^{\prime}$-chain from $s_{j}-\sigma_{j}$ to $\omega_{m_{j}+2}$, and a "symmetric" chain from $s_{j}+\sigma_{j}$ to $u_{j+1}-h$. (Here "symmetric" means that the points of the latter chain are symmetric about $s_{j}$ to the corresponding points of the former.) The two chains together with the element $\left(s_{j},\left[s_{j}-\sigma_{j}, s_{j}\right.\right.$ $\left.\left.+\sigma_{j}\right]\right) \in$ Sym $\subset H$ filling the gap between them extend our construction up to the point $u_{j+1}-h=\omega_{m_{j+1}-1}$. Put $\tau_{m_{j+1}}=u_{j+1}, \omega_{m_{j+1}}=u_{j+1}+h, \tau_{m_{j+1}+1}$ $=t_{j+1}, \omega_{m_{j+1}+1}=v_{j+1}-h, \tau_{m_{j+1}+2}=v_{j+1}, \omega_{m_{j+1}+2}=v_{j+1}+h$. Thus we have proceeded from step $j$ to step $j+1$ in our construction. Repeating the procedure, we extend the covering $\Omega$ to the whole interval $[a, b]$. It is seen directly from the construction that $\Omega$ has the required properties.

3.4. Proof of Saks-Henst ock lemma. Let $\eta>0$. Find gauges $\varphi_{j}$ on $\left[v_{j}, u_{j+1}\right]$ for $j=0,1, \ldots, k$ (denoting $v_{0}=a, u_{k+1}=b$ ) such that

$$
\left|(H) \int_{v_{j}}^{u_{j+1}} f d t-S\left(f, \Phi_{j}\right)\right|<\eta /(k+1)
$$

for every $\varphi_{j}$-fine $H$-covering $\Phi_{j}$ of $\left[v_{j}, u_{j+1}\right]$.

Let $\tilde{\delta}$ be a gauge on $[a, b]$ such that

$$
\begin{gathered}
\tilde{\delta}(t) \leqslant \delta(t) \quad \text { for } \quad t \in[a, b], \\
\tilde{\delta}(t)<\left|t-u_{j}\right| \quad \text { for } \quad t \neq u_{j}, \\
\tilde{\delta}(t)<\left|t-v_{j}\right| \quad \text { for } \quad t \neq v_{j}, \\
\tilde{\delta}(t) \leqslant \varphi_{j}(t) \quad \text { for } \quad t \in\left[v_{j}, u_{j+1}\right], \\
\tilde{\delta}\left(u_{j}\right)<\delta\left(t_{j}\right)-\left(t_{j}-u_{j}\right), \quad \tilde{\delta}\left(v_{j}\right)<\delta\left(t_{j}\right)-\left(v_{j}-t_{j}\right) ; \\
\max \left(\widetilde{\delta}\left(u_{j}\right), \tilde{\delta}\left(v_{j}\right)\right)<\xi=\xi\left(t_{j},\left[u_{j}, v_{j}\right]\right)
\end{gathered}
$$

(with $\xi$ from formula (1)), and

$$
\begin{aligned}
& \tilde{\delta}\left(u_{j}\right)\left|f\left(t_{j}\right)\right|<\eta / 2(k+1), \\
& \tilde{\delta}\left(v_{j}\right)\left|f\left(t_{j}\right)\right|<\eta / 2(k+1) .
\end{aligned}
$$

Let $\Omega$ be the partition from Lemma 3.3 (corresponding to the gauge $\tilde{\delta}$ instead of $\delta$ ). Since $\Omega$ is a $\delta$-fine $H$-covering of $[a, b]$ we have

$$
\left|(H) \int_{a}^{b} f(t) d t-S(f, \Omega)\right|<\varepsilon .
$$

Further, denote

$$
\begin{aligned}
\Phi_{j}=\left\{\left(\tau_{p},\left[\omega_{p-1}, \omega_{p}\right]\right) ; p\right. & \left.=m_{j}+2, m_{j}+3, \ldots, m_{j+1}\right\}, \\
j & =0,1, \ldots, k, m_{0}=-1, m_{k+1}=l .
\end{aligned}
$$

Then $\Phi_{j}$ is a $\varphi_{j}$-fine $H$-covering of $\left[v_{j}, u_{j+1}\right]$ and hence satisfies (10). Obviously we have 


$$
\begin{aligned}
\sum_{j=1}^{k}\left((H) \int_{u_{j}}^{v_{j}} f d t\right. & \left.-f\left(t_{j}\right)\left(v_{j}-u_{j}\right)\right)=(H) \int_{a}^{b} f d t-S(f, \Omega) \\
& -\sum_{j=0}^{k}\left((H) \int_{v_{j}}^{u_{j+1}} f d t-S\left(f, \Phi_{j}\right)\right)-\sum_{j=1}^{k} f\left(t_{j}\right)\left(v_{j}-\omega_{m_{j}+1}-u_{j}+\omega_{m_{j}}\right)
\end{aligned}
$$

and consequently,

$$
\left|\sum_{j=1}^{k}\left((H) \int_{u_{j}}^{v_{j}} f d t-f\left(t_{j}\right)\left(v_{j}-u_{j}\right)\right)\right|<\varepsilon+2 \eta .
$$

Since $\eta>0$ has been arbitrary, the proof is complete.

3.5. Remark. The assertion of the Saks-Henstock lemma can be modified to

$$
\sum_{j=1}^{k}\left|F\left(v_{j}\right)-F\left(u_{j}\right)-f\left(t_{j}\right)\left(v_{j}-u_{j}\right)\right| \leqslant 2 \varepsilon
$$

This is obtained by dividing the set of $\left(t_{j},\left[u_{j}, v_{j}\right]\right)$ in two groups according to the sign of the corresponding summand, and applying the lemma in the original form to each group separately.

Now we can prove a converse of Theorem 3.1.

3.6. Theorem. Let $f:[a, b] \rightarrow R$ be H-integrable, $d \in(a, b)$. If there exists a finite limit $\lim _{c>d} F(c)=q \in R$ for $c \in(a, d) \backslash E$, then $(H) \int_{a}^{d} f d t$ exists and equals $q$.

Proof. Let $\varepsilon>0$ and let $\delta$ be the gauge on $[a, b]$ corresponding to $\varepsilon$ and (H) $\int_{a}^{b} f d t$. Let $\tilde{\delta}$ be a gauge on $[a, b]$ such that

$$
\begin{array}{rll}
2|f(a)| \tilde{\delta}(d)<\varepsilon, & 2|f(d)| \tilde{\delta}(d)<\varepsilon, \\
|F(x)-q|<\varepsilon & \text { for any } & x \notin E, d-\tilde{\delta}(d)<x<d, \\
|F(x)|<\varepsilon & \text { for any } & x \notin E, a<x<a+\tilde{\delta}(a)
\end{array}
$$

(cf. Theorem 3.1),

$$
\tilde{\delta}(x) \leqslant \delta(x) \quad \text { for all } x \in[a, b] .
$$

Let $\Delta$ be a $\tilde{\delta}$-fine $H$-covering of $[a, d]$, where

$$
\Delta=\left\{\left(t_{j},\left[x_{j-1}, x_{j}\right]\right) ; j=1, \ldots, k\right\} .
$$

Find a $\tilde{\delta}$-fine modification $\Delta_{h}$ (cf. Remark 1.2) such that $x_{j}^{\prime}=$ $x_{j}+(-1)^{k-1-j} h \notin E$ for $j=1, \ldots, k, h>0$ and

$$
2 h \sum_{j=1}^{k}\left|f\left(t_{j}\right)\right|<\varepsilon
$$


By the Saks-Henstock lemma we have

$$
\left|\sum_{j=2}^{k-1}\left(f\left(t_{j}\right)\left(x_{j}^{\prime}-x_{j-1}^{\prime}\right)-(H) \int_{x_{j-1}^{\prime}}^{x_{j}^{\prime}} f d t\right)\right| \leqslant \varepsilon .
$$

Consequently, using (11), (12) and the properties of the gauge $\tilde{\delta}$ we obtain

$$
\begin{aligned}
\left|\sum_{j=1}^{k} f\left(t_{j}\right)\left(x_{j}-x_{j-1}\right)-q\right| \leqslant \varepsilon+\left|\sum_{j=1}^{k} f\left(t_{j}\right)\left(x_{j}^{\prime}-x_{j-1}^{\prime}\right)-q\right| \\
\leqslant \varepsilon+2 \tilde{\delta}(a)|f(a)|+\left|\sum_{j=2}^{k-1}\left[f\left(t_{j}\right)\left(x_{j}^{\prime}-x_{j-1}^{\prime}\right)-(H) \int_{x_{j-1}^{\prime}}^{x_{j}^{\prime}} f d t\right]\right| \\
+2 \widetilde{\delta}(d)|f(d)|+\left|(H) \int_{x_{i}^{\prime}}^{x_{k-1}^{\prime}} f d t-q\right| \\
\leqslant 4 \varepsilon+\left|F\left(x_{k-1}^{\prime}\right)-q\right|+\left|(H) \int_{a}^{x_{1}^{\prime}} f d t\right| \leqslant 6 \varepsilon,
\end{aligned}
$$

which proves the theorem.

The next theorem strengthens the result on continuity of the function $F$, asserting that it has a derivative equal to $f$ almost everywhere in $[a, b]$. The symbol $m(M)$ stands for the Lebesgue measure of the set $M$.

3.7. ThEOREM. There is a set $M \subset[a, b], m(M)=0$, such that for every $\varepsilon>0$ and $t \in[a, b] \backslash M$ there is $\vartheta=\vartheta(t)>0$ such that

$$
|F(y)-F(x)-f(t)(y-x)|<\varepsilon|y-x|
$$

for every $x, y$ such that

$$
(t,[x, y]) \in H, \quad[x, y] \subset B(t, \vartheta(t)), \quad x, y \notin E .
$$

Proof. For $\vartheta>0, t \in[a, b] \backslash E$ define

$$
\begin{array}{ll}
\Phi_{\vartheta}(t)=\inf (F(y)-F(x)) /(y-x), & \Phi_{*}(t)=\sup _{\vartheta} \Phi_{\vartheta}(t), \\
\Phi^{\vartheta}(t)=\sup (F(y)-F(x)) /(y-x), & \Phi^{*}(t)=\inf _{\vartheta} \Phi^{\vartheta}(t),
\end{array}
$$

where the infimum or supremum is taken over all $x, y$ satisfying (14). Denote

$$
\begin{aligned}
& P_{n}=\left\{t \in(a, b) ; \Phi_{*}(t) \leqslant f(t)-n^{-1}\right\}, \\
& Q_{n}=\left\{t \in(a, b) ; \Phi^{*}(t) \geqslant f(t)+n^{-1}\right\}, \\
& M=\bigcup_{n=1}^{\infty}\left(P_{n} \cup Q_{n}\right) .
\end{aligned}
$$

If $m(M)=0$, the theorem holds. Assume $m_{e}(M)>0$. Then there exists, say, an index $p$ such that $m_{e}\left(P_{p}\right)=\sigma>0\left(m_{e}\right.$ denotes the outer Lebesgue measure; the proof is analogous if $m_{e}\left(Q_{q}\right)>0$ for some $\left.q\right)$. 
Choose $0<\varepsilon<\sigma / 4 p$ and find the gauge $\delta$ corresponding to $\varepsilon$ by Definition 2.1. For $t \in P_{p}$ set

$$
\begin{aligned}
\mathscr{S}(t)=\{(x, y) \subset[a, b] ; x, y \notin E,(t,[x, y]) \in H,[x, y] \in B(t, \delta(t)), \\
\left.(F(y)-F(x))(y-x)^{-1}<f(t)-1 / 2 p\right\} .
\end{aligned}
$$

Then $\bigcup_{t \in P_{p}} \mathscr{S}(t)$ covers $P_{p}$ in the sense of Vitali; hence there exists its finite disjoint subsystem of $\left(x_{i}, y_{i}\right), i=1, \ldots, r$, such that

$$
\sum_{i=1}^{r}\left(y_{i}-x_{i}\right) \geqslant \sigma / 2 \text {. }
$$

We may apply the Saks-Henstock lemma to this subsystem, which yields

$$
\left|\sum_{i=1}^{r}\left(F\left(y_{i}\right)-F\left(x_{i}\right)-f\left(t_{i}\right)\left(y_{i}-x_{i}\right)\right)\right| \leqslant \varepsilon<\sigma / 4 p
$$

on the other hand, from the definition of $\mathscr{S}(t)$ we have

$$
\left|\sum_{i=1}^{r}\left(F\left(y_{i}\right)-F\left(x_{i}\right)-f\left(t_{i}\right)\left(y_{i}-x_{i}\right)\right)\right|>\sum_{i=1}^{r} \frac{1}{2 p}\left(y_{i}-x_{i}\right) \geqslant \frac{\sigma}{4 p},
$$

a contradiction.

3.8. THEOREM. Let $F$ be defined as above, put $F(t)=F(a)$ for $t<a$ and $F(t)=F(b)$ for $t>b$. Let $C \subset[a, b], m(C)=0$. Then for every $\varepsilon>0$ there is a gauge $\delta$ on $C$ such that for any finite system $\left\{\left(\tau_{j},\left[\xi_{j}, \eta_{j}\right]\right) ; j=1, \ldots, r\right\} \subset H$ such that $\tau_{j} \in C,\left[\xi_{j}, \eta_{j}\right] \subset B\left(\tau_{j}, \delta\left(\tau_{j}\right)\right) ; \xi_{j}, \eta_{j} \notin E$ and the intervals $\left[\xi_{j}, \eta_{j}\right] d o$ not overlap, the inequality

$$
\sum_{j=1}^{r}\left|F\left(\eta_{j}\right)-F\left(\xi_{j}\right)\right|<\varepsilon
$$

holds.

Proof. Denote

$$
C_{n}=\{t \in C ; n-1 \leqslant|f(t)|<n\} .
$$

There exist open sets $G_{n} \supset C_{n}, m\left(G_{n}\right)<\varepsilon_{n}=\varepsilon\left(3 \cdot 2^{n} n\right)^{-1}$; for $t \in C_{n}$ choose $\delta_{1}(t)$ such that $B\left(t, \delta_{1}(t)\right) \subset G_{n}$. Since $\left[\xi_{j}, \eta_{j}\right]$ do not overlap, we have

$$
\sum_{j=1}^{r}\left|f\left(\tau_{j}\right)\right|\left(\eta_{j}-\xi_{j}\right)=\sum_{n=1}^{\infty} \sum_{\tau_{j} \in C_{n}}\left|f\left(\tau_{j}\right)\right|\left(\eta_{j}-\xi_{j}\right) \leqslant \sum_{n=1}^{\infty} n m\left(G_{n}\right)<\varepsilon / 3
$$

For $\varepsilon / 3$ find the gauge $\delta_{2}$ from Definition 2.1 and put $\delta(t)=\min \left(\delta_{1}(t), \delta_{2}(t)\right)$ for $t \in C, \delta(t)=\delta_{2}(t)$ otherwise. Using the modified version of the Saks-Henstock lemma from Remark 3.5 we obtain

$$
\sum_{j=1}^{r}\left|F\left(\eta_{j}\right)-F\left(\xi_{j}\right)-f\left(\tau_{j}\right)\left(\eta_{j}-\xi_{j}\right)\right|<\frac{2}{3} \varepsilon
$$


that is,

$$
\sum_{j=1}^{r}\left|F\left(\eta_{j}\right)-F\left(\xi_{j}\right)\right|<\frac{2}{3} \varepsilon+\sum_{j=1}^{r}\left|f\left(\tau_{j}\right)\right|\left(\eta_{j}-\xi_{j}\right)<\varepsilon
$$

by inequality (16).

The next theorem shows that the properties from the two preceding theorems characterize the indefinite $H$-integral.

3.9. THEOREM. Let $E$ be an at most countable subset of $[a, b]$, let $f:[a, b] \rightarrow R, F:[a, b] \backslash E \rightarrow R$. Extend $F$ by $F(t)=F(a)$ for $t<a, F(t)=$ $F(b)$ for $t>b$.

Assume that

(i) for almost all $t$ and all $\varepsilon>0$ there exists $\vartheta(t)$ such that (13) holds for all $x$, $y$ satisfying (14);

(ii) if $C \subset[a, b], m(C)=0$, then for every $\varepsilon>0$ there is a gauge $\delta$ on $C$ such that (15) holds provided $\tau_{j}, \xi_{j}, \eta_{j}$ satisfy the assumptions of Theorem 3.8 .

Then $(H) \int_{a}^{b} f d t$ exists and equals $F(b)-F(a)$.

Proof. Let $C_{1}$ be the set of $t \in[a, b]$ for which (i) is not fulfilled, $C=C_{1} \cup E$. Then $m(C)=0$. Find $\delta$ from (ii) and define $\delta_{1}(t)=\min (\delta(t), \vartheta(t))$ for $t \in C, \delta_{1}(t)=\vartheta(t)$ otherwise. Let $\varepsilon>0$ and let $\Delta$ be a $\delta_{1}$-fine $H$-partition of $[a, \mathrm{~b}]$,

$$
\Delta=\left\{\left(t_{j},\left[x_{j}, y_{j}\right]\right) ; j=1, \ldots, k\right\} .
$$

Similarly as in the proof of Theorem 3.6 , modify $\Delta$ so that $F\left(x_{j}^{\prime}\right), F\left(y_{j}^{\prime}\right)$ are defined for all $j$ 's and $S(f, \Delta)$ changes only by $\varepsilon$ (cf. Remark 1.2 and inequality (11)). Then

$$
(H) \int_{a}^{b} f d t=\sum_{j=1}^{k}\left[F\left(y_{j}^{\prime}\right)-F\left(x_{j}^{\prime}\right)\right]
$$

hence

$$
\begin{aligned}
\left|(H) \int_{a}^{b} f d t-S\left(f, \Delta_{h}\right)\right| \leqslant \sum_{t_{j} \notin C} \mid F\left(y_{j}^{\prime}\right) & -F\left(x_{j}^{\prime}\right)-f\left(t_{j}\right)\left(y_{j}^{\prime}-x_{j}^{\prime}\right) \mid \\
& +\sum_{t_{j} \in C}\left|F\left(y_{j}^{\prime}\right)-F\left(x_{j}^{\prime}\right)\right|+\sum_{i_{j} \in C}\left|f\left(t_{j}\right)\right|\left(y_{j}^{\prime}-x_{j}^{\prime}\right) .
\end{aligned}
$$

Using (i), (ii) and estimating the last sum as in the proof of the preceding theorem we conclude

$$
\left|\int_{a}^{b} f d t-S(f, \Delta)\right| \leqslant \text { const } \cdot \varepsilon
$$

which completes the proof. 


\section{References}

[1] K. J a cobs, Measure and Integral, Academic Press, New York-San Francisco-London 1978.

[2] J. Kurzweil, Generalized Ordinary Differential Equations and Continuous Dependence on a Parameter, Czech. Math. J. 7 (82) (1957), 568-583.

[3] -. The Integral as a Limit of Integral Sums, Jahrbuch Überblicke Mathematik (1984), 105-136, Bibliographisches Institut AG(1984).

[4] - and J. Jarník, On some extensions of the Perron integral on one-dimensional intervals. An approach by integral sums fulfilling a symmetry condition, Functiones et Approximatio 17(1987), 49-55.

MATHEMATICAL INSTITUTE. CZECHOSLOVAK ACADEMY OF SCIENCES

PRAGUE. CZECHOSLOVAKIA

Reçu par la Rédaction le 26.05.1988 